\title{
Controversies surrounding peripheral cannabinoid receptor 1 in fatty liver disease
}

\author{
Beste Mutlu ${ }^{1,2}$ and Pere Puigserver, ${ }^{1,2}$ \\ 'Department of Cancer Biology, Dana-Farber Cancer Institute, Boston, Massachusetts, USA. ²Department of Cell Biology, Harvard Medical School, Boston, Massachusetts, USA.
}

\begin{abstract}
Cannabinoid receptor 1 (CB-1) antagonists are potential candidates for treating obesity and metabolic complications. Despite clear metabolic benefits, unwanted side effects in the brain pose issues for patients. With the hope of overcoming this obstacle, CB-1 in peripheral tissues has become a potential drug target. Previous studies had suggested that liver CB-1 would be an excellent target to prevent development of nonalcoholic steatohepatitis (NAFLD). However, in this issue of the $J C I$, Wang et al. showed that CB-1 was barely detectable in the liver and deletion of CB-1 in hepatocytes provided no metabolic benefits against NAFLD. These contradictory results raise substantial concerns about the potential benefits of peripheral CB-1 blockers against NAFLD.
\end{abstract}

\section{Sources of liver fat in metabolic diseases}

NAFLD is a continuum of liver pathologies, usually seen in overweight or obese people, that can be described in three progressive stages: (a) fat accumulation in the liver (hepatic steatosis), (b) nonalcoholic steatohepatitis (NASH) associated with inflammation and fibrosis, and ultimately (c) cirrhosis and hepatocellular carcinoma (1). Excessive fat deposition into the liver, the earliest metabolic driver of NAFLD/ $\mathrm{NASH}$, can occur via several mechanisms. One important source of liver fat is lipolysis from adipose tissue, which releases free fatty acids (FFAs) into circulation that can be taken up by the liver. In healthy individuals, fasting promotes lipolysis and is suppressed by insulin after feeding. In the context of type 2 diabetes and insulin resistance, unrestrained lipolysis leads to excess circulating FFAs that get deposited into the liver (2). Another major contributor is de novo lipogenesis (DNL) in the liver, a pathway that produces FA chains from acetyl-CoA subunits by utilizing glucose and other substrates (3). DNL is upregulated in insulin resistance linked to unrestrained hepatic glucose production (HGP) and impaired glucose uptake in skeletal muscle, providing glucose and other substrates for DNL. Thus, insulin resistance causes an increase in HGP, exacerbating hyperglycemia, but does not further suppress DNL, contributing to the development of fatty liver. Dietary fat intake is a third source of liver fat accumulation.

It is not clear whether targeting one of these pathways is more advantageous compared with others in the prevention and treatment of NAFLD. A recent study has shown that approximately $47 \%$ of FAs that comprise triglycerides (TGs) in the liver come from FFAs in the blood, $38 \%$ from DNL, and 15\% from the diet (ref. 4 and Figure 1). It has been proposed that increased DNL is the main culprit in NAFLD (5). Although DNL is a substantial source of lipids that increases in subjects with NAFLD, nocturnal plasma FFA levels also increase with NAFLD (6). Thus, liver DNL is not the only pathway that may be targeted for the treatment of NAFLD. Esler et al. (7) review many different treatment

Related Article: https://doi.org/10.1172/JCl152242

Conflict of interest: The authors have declared that no conflict of interest exists.

approaches, including directly modulating lipid metabolism in the liver through the enzymes acetyl-CoA carboxylase (ACC), fatty acid synthase (FAS), and diacylglycerol acyltransferase 2 (DGAT2). They also highlight a strategy to alter the delivery of FFAs from adipose tissue to the liver by targeting insulin resistance and/or adipose metabolism (7). Indeed, activation of adipocyte $G_{i}$ signaling to suppress lipolysis leads to decreased plasma FFA levels and liver TG levels (8). Weight loss through dietary restriction or other approaches is also a valid strategy against NAFLD (9).

\section{CB-1 as a potential drug target for NAFLD}

$G$ protein-coupled cannabinoid receptors and their ligands play a critical role in regulating energy homeostasis. In the early years of the field, research focused on the regulation of appetite by endocannabinoids that act via $\mathrm{CB}-1$ in the hypothalamus (10). Although CB-1 antagonists looked promising for the treatment of obesity and its metabolic complications, unwanted neuropsychiatric effects led to their withdrawal from the market. For instance, the first CB-1 antagonist, rimonabant, caused weight loss and improved cardiometabolic parameters in overweight people (11), but also increased anxiety and suicidal ideation (12). Later, the discovery of functional CB-1 in peripheral tissues ushered the development of antagonists that are unable to cross the bloodbrain barrier, with the hope of reaping the metabolic benefits by targeting peripheral tissues without detrimental side effects (13). Many studies that are reviewed by Cinar et al. (13) highlight hepatocytes, adipose tissue, skeletal muscle, and pancreatic $\beta$ cells as potential peripheral targets for CB-1 antagonists.

\section{Liver CB-1 is not a good peripheral target for NAFLD}

Previous reports suggested that the liver would be a good peripheral tissue for 


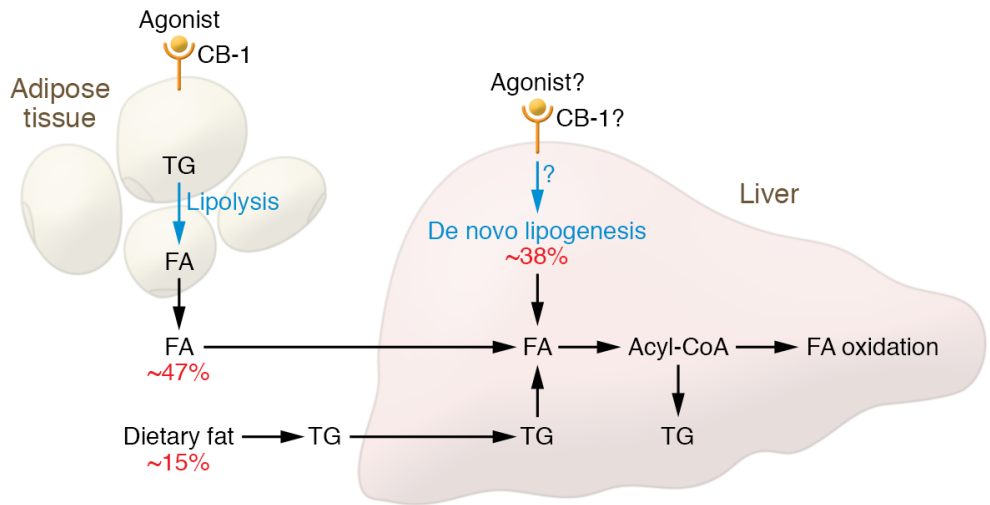

Relative contribution to liver TG during NAFLD

Downtsream effects of peripheral CB-1 signaling

Figure 1. Source of liver fats during NAFLD and the role of CB-1 signaling in liver and adipose tissue. Free fatty acids (FFAs) produced by lipolysis in adipose tissue, de novo lipogenesis (DNL) in the liver, and dietary lipids are major sources (approximately $47 \%, 38 \%$, and $15 \%$, respectively) of FAs that comprise triglycerides (TG) in the liver. Activation of cannabinoid receptor 1 (CB-1) signaling by agonists in the liver was previously shown to increase DNL (14). Wang et al. (17) dispute the presence of CB-1 in the liver and downstream metabolic effects. However, it is possible that blockade of CB-1 signaling in adipose tissue may decrease lipolysis and FFA release, thereby protecting against NAFLD.

a pharmaceutical intervention against NAFLD (Figure 1). CB-1 is expressed in the liver, and its activation in hepatocytes by an agonist leads to increased DNL (14). Whole-body and liver-specific CB-1knockout mice both show less steatosis on a high-fat diet (HFD), and CB-1 agonist treatment fails to increase DNL in these animals (15), highlighting the regulation of liver DNL through CB-1. Recapitulating the effects seen by Osei-Hyiaman et al., another study showed that liver-specific CB-1-knockout mice have decreased insulin resistance on an HFD (16). However, in this issue of the JCI, Wang et al. raise concerns about the reproducibility of the effects seen in hepatocyte-specific CB-1knockout mice (17). Wang et al. reveal that deletion of CB-1 in hepatocytes did not alter DNL or insulin resistance, or the development of NAFLD in response to an HFD, and was not protective against carbon tetrachloride-induced fibrosis (17).

Wang et al. convincingly show that the liver is unlikely to be a relevant target for CB-1 antagonists (17). Their elegant and thorough single-cell gene expression studies with mouse cells show that CB-1 was barely detectable in the liver tissue among the different cell types analyzed, consistent with a previous report (18). Analysis of liver samples from human patients with NAFLD/NASH showed minimal expression of this receptor. This result contra- dicts previous studies that claim that liver samples from NAFLD patients have a 34.2fold increase in CB-1 expression compared with controls (16). Why this discrepancy? It is possible that CB-1 in the liver in NAFLD patients is regulated by unknown factors, e.g., the degree of liver fibrosis (19) or gut microbiota (20). Since the microbiome composition of mice is affected by housing conditions, this variability might explain the discrepancy between studies from different laboratories.

\section{Is adipose tissue CB-1 a feasible target in NAFLD?}

An important question that remains unanswered is whether peripheral CB-1 antagonists can still be useful in the treatment of NAFLD. Nimacimab, a peripheral negative-allosteric modulating antibody targeting CB-1, has completed a phase Ib study for NAFLD treatment. Success may still be achieved through off-target effects or other peripheral tissues. For the latter, adipose tissue is a plausible candidate. Lipolysis in the adipose tissues releases FFAs into the bloodstream and is a major source of liver fat. CB-1 is highly expressed in adipose tissues both in mice $(18,21,22)$ and humans (23), making it a feasible pharmaceutical target. Moreover, CB-1 expression in adipocytes is upregulated in obese rats (22). Activation of adipocyte $C B-1$ leads to increased lipolysis (ref. 24 and Figure 1) and its adipo- cyte-specific deletion in mice is sufficient to prevent diet-induced obesity (25). Until these studies are disproven using pharmacological and genetic approaches, there is still hope for peripheral CB-1 inhibitors to treat obesity and NAFLD.

\section{Acknowledgments}

$\mathrm{PP}$ is financially supported by $\mathrm{NIH}$ grants R01 DK089883, R01 DK081418, R01 DK117655, R01 CA181217, 9R56 AG074527, and R01 GM121452, and a Claudia Adams Barr Award. BM is supported by NIH grant F32 GM136019-01A1.

Address correspondence to: Pere Puigserver or Beste Mutlu, Dana-Farber Cancer Institute, 360 Brookline Ave., LC 6213, Boston, Massachusetts 02115-5450, USA. Phone: 617.582.7977; Email: pere puigserver@dfci.harvard.edu (PP); Email: beste_mutlu@dfci.harvard.edu (BM).

1. Bence KK, Birnbaum MJ. Metabolic drivers of non-alcoholic fatty liver disease. Mol Metab. 2021;50:101143.

2. Guilherme A, et al. Adipocyte dysfunctions linking obesity to insulin resistance and type 2 diabetes. Nat Rev Mol Cell Biol. 2008;9(5):367-377.

3. Alves-Bezerra M, Cohen DE. Triglyceride metabolism in the liver. Compr Physiol. 2017;8(1):1-8.

4. Smith GI, et al. Insulin resistance drives hepatic de novo lipogenesis in nonalcoholic fatty liver disease. JClin Invest. 2020;130(3):1453-1460.

5. Solinas G, et al. De novo lipogenesis in metabolic homeostasis: More friend than foe? Mol Metab. 2015;4(5):367-377.

6. Lambert JE, et al. Increased de novo lipogenesis is a distinct characteristic of individuals with nonalcoholic fatty liver disease. Gastroenterology. 2014;146(3):726-735.

7. Esler WP, Bence KK. Metabolic targets in nonalcoholic fatty liver disease. Cell Mol Gastroenterol Hepatol. 2019;8(2):247-267.

8. Wang L, et al. Adipocyte $G_{i}$ signaling is essential for maintaining whole-body glucose homeostasis and insulin sensitivity. Nat Commun. 2020;11(1):2995

9. Chalasani $\mathrm{N}$, et al. The diagnosis and management of nonalcoholic fatty liver disease: practice guidance from the American Association for the Study of Liver Diseases. Hepatology. 2018;67(1):328-357.

10. Di Marzo V, et al. Leptin-regulated endocannabinoids are involved in maintaining food intake. Nature. 2001;410(6830):822-825.

11. Després JP, et al. Effects of rimonabant on metabolic risk factors in overweight patients with dyslipidemia. NEngl JMed. 2005;353(20):2121-2134.

12. Christensen R, et al. Efficacy and safety of the weight-loss drug rimonabant: a meta-analysis of randomised trials. Lancet. 2007;370(9600):1706-1713. 
13. Cinar R, et al. The therapeutic potential of second and third generation $\mathrm{CB}_{1} \mathrm{R}$ antagonists. Pharmacol Ther. 2020;208:107477.

14. Osei-Hyiaman D, et al. Endocannabinoid activation at hepatic $\mathrm{CB} 1$ receptors stimulates fatty acid synthesis and contributes to diet-induced obesity. J Clin Invest. 2005;115(5):1298-1305.

15. Osei-Hyiaman D, et al. Hepatic CB1 receptor is required for development of diet-induced steatosis, dyslipidemia, and insulin and leptin resistance in mice. J Clin Invest. 2008;118(9):3160-3169.

16. Liu J, et al. Hepatic cannabinoid receptor-1 mediates diet-induced insulin resistance via inhibition of insulin signaling and clearance in mice. Gastroenterology. 2012;142(5):1218-1228.

17. Wang S, et al. Cannabinoid receptor 1 signaling in hepatocytes and stellate cells does not contribute to NAFLD. JClin Invest. 2021;131(21):e152242.

18. Tang Y, et al. Beneficial metabolic effects of CB1R anti-sense oligonucleotide treatment in diet-induced obese AKR/J mice. PLoS One. 2012;7(8):e42134.

19. Dai E, et al. Hepatic expression of cannabinoid receptors $\mathrm{CB} 1$ and $\mathrm{CB} 2$ correlate with fibrogenesis in patients with chronic hepatitis B. Int J Infect Dis. 2017;59:124-130.

20. Lang S, Schnabl B. Microbiota and fatty liver disease-the known, the unknown, and the future. Cell Host Microbe. 2020;28(2):233-244.

21. Vida M, et al. CB1 blockade potentiates down-regulation of lipogenic gene expression in perirenal adipose tissue in high carbohydrate diet-induced obesity. PLoS One. 2014;9(2):e90016

22. Bensaid M, et al. The cannabinoid CB1receptor antagonist SR141716 increases Acrp30 mRNA expression in adipose tissue of obese $\mathrm{fa} / \mathrm{fa}$ rats and in cultured adipocyte cells. Mol Pharmacol. 2003;63(4):908-914.

23. Uhlen M, et al. Proteomics. Tissue-based map of the human proteome. Science. 2015;347(6220):1260419.

24. Muller T, et al. Overactivation of the endocannabinoid system alters the antilipolytic action of insulin in mouse adipose tissue. Am J Physiol Endocrinol Metab. 2017;313(1):E26-E36.

25. Ruiz De Azua I, et al. Adipocyte cannabinoid receptor CB1 regulates energy homeostasis and alternatively activated macrophages. J Clin Invest. 2017;127(11):4148-4162. 